\title{
Motivos de abandono do tratamento antirrábico humano pós-exposição em Porto Alegre (RS, Brasil)
}

\author{
Abandon reasons of post-exposure human anti-rabies treatment \\ in Porto Alegre (RS, Brazil)
}

Rejane Dias Veloso ${ }^{1}$

Denise Rangel Ganzo de Castro Aerts ${ }^{1}$

Liane Oliveira Fetzer ${ }^{2}$

Celso Bittencourt dos Anjos ${ }^{3}$

José Carlos Sangiovanni ${ }^{4}$

${ }^{1}$ Programa de

Pós-Graduação em Saúde

Coletiva, Universidade

Luterana do Brasil. Av.

Farroupilha 8001, prédio 14,

sala 226, Bairro São José.

92425-900 Canoas RS.

dv-rejane@uol.com.br

${ }^{2}$ Equipe de Vigilância de

Doenças Transmissíveis,

Núcleo de Imunizações,

Coordenadoria Geral de

Vigilância em Saúde,

Secretaria Municipal de

Saúde de Porto Alegre.

${ }^{3}$ Centro Estadual de

Vigilância em Saúde do Rio

Grande do Sul.

${ }^{4}$ Centro de Controle de

Zoonoses, Coordenadoria

Geral de Vigilância em

Saúde, Secretaria Municipal

de Saúde de Porto Alegre.
Abstract In Brazil, rabies is an endemic disease with a fatality rate of $100 \%$. The number of cases has decreased, but the number of cases for treatment after exposure and treatment dropout is still high. This study investigated the causes of antirabies treatment abandon, after exposure, in Porto Alegre (RS, Brazil), from July to December 2006. A case series was designed. Two hundred and eighty abandon cases were selected through randomized systematic sampling, out of 962 registered in Sinan. Data was collected in people's homes through interviews, by means of a questionnaire. According to the interviewees, $66.4 \%$ concluded the prescribed number of vaccines. This information was not registered in Sinan. Among the subjects confirmed of abandoning the treatment (94/280), 24.5\% reported that they thought it was not necessary to complete the treatment, while $13.8 \%$ felt that they did not receive clear guidelines about what to do. Health services attempted to contact absents in only $19.2 \%$ of the cases. Data entered in Sinan present failures. These occurred because patients started treatment at one health service and continued in a different one. As a consequence, information about the conclusion of the treatment was not entered into the system. Key words Rabies, Anti-rabies vaccination, Epidemiologic profile
Resumo A raiva é uma doença endêmica no Brasil, com letalidade de $100 \%$. O número de casos tem diminuído, porém o de tratamento pós-exposição continua elevado, assim como o de abandono. O objetivo do estudo foi investigar as causas do abandono do tratamento antirrábico humano pós-exposição em Porto Alegre (RS), no segundo semestre de 2006. Foi utilizado o delineamento de série de casos, sendo selecionados 280 casos por amostragem aleatória sistemática entre os 962 registrados no Sinan como abandono de tratamento. Os dados foram coletados em entrevistas domiciliares, utilizando-se questionário específico. Segundo os entrevistados, 66,4\% concluíram o número de vacinas prescritas, não estando esses dados registrados no Sinan. Entre aqueles que foram confirmados como abandono (94/280), 24,5\% referiram não ter considerado necessário completá-lo, e 13,8\% não se sentiram orientados sobre como proceder. Somente em 19,1\% dos casos houve a busca ativa dos faltosos pelos serviços de saúde. O registro no Sinan apresenta falhas. Estas ocorrem devido ao fato de o paciente iniciar o tratamento em um serviço de saúde e dar continuidade em outro, não havendo retroalimentação do sistema com dados sobre a sua conclusão.

Palavras-chave Raiva, Vacinas contra raiva, Perfil epidemiológico 


\section{Introdução}

A raiva é uma antropozoonose transmitida ao homem pela inoculação do vírus presente na saliva e nas secreções do animal infectado, pela mordedura, arranhadura ou lambedura da pele lesada ou mucosa, por aerossóis ou transplante de córnea ${ }^{1,2}$. Os mamíferos são os únicos animais suscetíveis à doença $a^{1,3}$.

A raiva continua a ser um problema de saúde pública, não em razão do número de casos, que têm reduzido ao longo dos anos, mas devido a sua letalidade de $100 \%$. Além disso, o impacto emocional, o sofrimento e a ansiedade das pessoas agredidas, temendo por contrair a doença ${ }^{4}$, devem ser levados em conta.

Não existe tratamento específico para a raiva. Após o início dos sintomas, a conduta é sintomática, visando apenas diminuir o sofrimento do paciente $e^{3,5}$. A profilaxia para indivíduos potencialmente infectados pelo vírus deve ser executada rigorosamente ${ }^{3,5}$, sendo essa a abordagem primária para a raiva ${ }^{2}$. Por essa razão, é considerada uma urgência médica², devendo o tratamento ser instituído o mais rápido possível.

No Brasil, sempre que ocorrer agressão animal, deve ser realizada a anamnese completa, obtendo todas as informações para a correta indicação do tratamento e registro dos dados na Ficha de Atendimento Antirrábico Humano, do Sistema de Informação de Agravos de Notificação (Sinan $)^{6}$. Logo após a agressão, deve ser feita a limpeza da lesão e, quando necessária, a administração da vacina contra a raiva, associada ou não ao uso de soro ou imunoglobulina humana ${ }^{3}$.

Desde 2002, é utilizada a vacina de cultivo celular ${ }^{7}$. Há dois esquemas para tratamento com o uso dessa vacina, de acordo com as condições do animal agressor e o tipo de exposição (agressões leves ou graves): duas doses, sendo uma administrada no dia da agressão e outra no terceiro dia; ou cinco doses, sendo administradas no dia da agressão, no $3^{\circ}$, no $7^{\circ}$, no $14^{\circ}$ e no $28^{\circ}$ dias ${ }^{1}$.

O soro deve ser utilizado em casos de acidentes graves em que o animal (cão ou gato) tenha desaparecido, morrido ou se tornado raivoso; em casos de animal clinicamente suspeito da raiva no momento da agressão; ou acidentes graves de animais silvestres ou de produção․ Sua aplicação é necessária para conferir anticorpos passivos ao indivíduo até que os anticorpos vacinais estejam presentes. Mortes por raiva têm sido atribuídas à falta do uso do soro, apesar do tratamento vacinal ${ }^{8}$.

Ao prescrever o tratamento profilático, devese ter presente que tanto o soro quanto a vacina podem originar complicações ${ }^{4}$. A indicação desnecessária, além de expor o paciente a eventos adversos, constitui-se em um desperdício dos recursos públicos, refletindo na qualidade do sistema de saúde 3 .

Os pacientes devem receber orientações quanto à gravidade da agressão, necessidade do tratamento, continuidade dele, cuidados com o ferimento, observação do animal agressor, importância da vacinação e, no caso de cães, de sua domicialização ${ }^{9}$.

O número de casos de raiva tem diminuído desde a década de 80 , porém o número de tratamentos pós-exposição continua elevado ${ }^{10}$, assim como o número de abandonos de tratamento. A não conclusão do tratamento prescrito não garante a imunização, podendo comprometer a sobrevida do paciente ${ }^{3}$.

A análise dos dados referentes aos tratamentos antirrábicos humanos possibilita a avaliação e o aprimoramento dos serviços de assistência e de vigilância epidemiológica de uma região ${ }^{11}$. Assim, o objetivo deste estudo foi investigar os motivos referidos pelos indivíduos agredidos para o abandono do tratamento antirrábico humano pós-exposição em Porto Alegre (RS), no segundo semestre de 2006.

\section{Material e métodos}

O delineamento utilizado foi a série de casos. No período de interesse, segundo semestre de 2006, ocorreram 2.223 atendimentos antirrábicos humanos pós-exposição, segundo dados disponíveis no Sinan. Entre esses, 1.737 receberam indicação de vacina e $962(55,4 \%)$ foram considerados como tendo abandonado o tratamento, uma vez que não havia registro da sua conclusão na ficha de atendimento.

Para o cálculo do tamanho da amostra, utilizou-se uma frequência esperada de $50 \%$, erro máximo de $\pm 5 \%$ e nível de significância de 0,05 , totalizando 275 indivíduos. A amostra foi estratificada segundo as nove unidades de referência para atendimento antirrábico, sendo os casos selecionados por meio de amostragem sistemática. Ao final do processo, haviam sido selecionados 280 casos. A fonte dos dados foi o banco do Sinan fornecido pela Coordenadoria Geral de Vigilância em Saúde (CGVS) da Secretaria Municipal de Saúde de Porto Alegre, Rio Grande do Sul (SMS/POA/RS).

Os sujeitos selecionados foram buscados por meio de visita domiciliar. Durante esse processo, 65 $(23,2 \%)$ indivíduos não foram localizados em ra- 
zão de problemas com o endereço (dez moravam em outro município e 55 eram desconhecidos dos moradores do endereço), um havia falecido $(0,4 \%)$ e nove $(3,2 \%)$ recusaram-se a participar. Com isso, foram substituídos 75 (26,8\%) indivíduos. O critério de seleção para a substituição foi o sorteio entre o sujeito imediatamente anterior ou posterior do caso inicialmente selecionado.

Foram realizadas 280 entrevistas com o auxílio de questionário construído especialmente para esse fim. As questões fechadas eram relativas ao serviço no qual o paciente iniciou o tratamento, se o tratamento foi concluído ou não, número de doses recebidas e se o paciente foi procurado por algum profissional de saúde do serviço no qual estava realizando o tratamento em razão de tê-lo interrompido.

Para a identificação do número de doses recebidas e confirmação da situação de abandono, foi solicitada a apresentação da carteira de vacina. Caso ela não fosse localizada, era solicitado ao entrevistado que referisse o número de doses prescritas e realizadas. A avaliação final sobre conclusão ou abandono do tratamento foi realizada compatibilizando-se o registro na carteira de vacina com o número de doses prescritas na ficha de atendimento antirrábico do Sinan ou comparando-se o número de doses referidas como realizadas com o número de doses prescritas na ficha.

A idade e o sexo do paciente foram coletados da ficha do Sinan e confirmados na entrevista. A idade foi categorizada segundo o ciclo de vida em: crianças (<6 anos e entre 6 e 12 anos), adolescentes (13 a 19 anos), adultos (20 a 59 anos) e idosos (> 60 anos).

Para as questões abertas, referentes ao motivo da procura pelo atendimento; motivo da troca de serviço, caso tivesse ocorrido; importância da vacinação e motivo da não conclusão do tratamento, procedeu-se à análise de conteúdo temática ${ }^{12}$.

Com vistas à análise da adequação da indicação do tratamento, foram utilizadas como referência as Normas Técnicas para Tratamento Profilático Antirrábico Humano ${ }^{1}$. Segundo estas, para caninos e felinos é considerado o estado de saúde do animal no momento da agressão, a possibilidade de observação dele, as características do ferimento e situação epidemiológica do município onde ocorreu a agressão. Em casos de contato com quirópteros ou agressão por animais de produção, a vacina deve ser sempre indicada ${ }^{1}$.

Cada caso foi, inicialmente, avaliado por um veterinário da equipe de pesquisa. Nas situações de discordância entre a prescrição do profissio- nal de saúde e o preconizado pelas Normas Técnicas, um técnico da CGVS atuou como o segundo avaliador. Além disso, um terceiro avaliador, especialista em raiva, revisou o caso, sendo considerado como o padrão ouro. Os tratamentos foram classificados em adequadamente e inadequadamente indicados.

Por fim, os dados quantitativos foram analisados utilizando-se a estatística descritiva, e as associações de interesse foram testadas com o auxílio do teste do Qui-quadrado de associação e nível de significância de 0,05 .

O projeto foi aprovado pelo Comitê de Ética e Pesquisa da Universidade Luterana do Brasil, tendo a CGVS/SMS/POA consentido no repasse dos dados e os entrevistados assinado o termo de consentimento.

\section{Resultados}

Entre os 280 indivíduos considerados como tendo abandonado o tratamento vacinal, 52,1\% eram do sexo masculino. A faixa etária que concentrou a maioria dos casos de interrupção de tratamento foi a dos adultos (50,8\%), seguida das crianças entre 6 e 12 anos (Tabela 1).

A distribuição dos casos de abandono, segundo sexo e faixa etária, foi comparada com o perfil de atendimentos antirábicos em Porto Alegre no mesmo período, não tendo apresentado diferenças estatisticamente significativas.

Em relação aos motivos da procura pelo atendimento, 45,0\% referiram ser para tratar o ferimento, 30,7\% para receber vacina antirrábica e 14,0\% em razão da gravidade da doença. Além desses, $4,3 \%$ o fizeram por razões não relacionadas à raiva, como para vacinação antitetânica; tratamento de problemas de saúde preexistentes (diabetes, hipertensão, baixa imunidade) ou situações relacionadas a acidentes de trabalho.

Entre os 280 casos estudados, 57 deles (20,4\%) recorreram ao serviço do Pronto-Socorro Municipal para o tratamento do ferimento. Nesse local, não é preenchida a ficha de atendimento antirrábico nem realizada a vacinação. Em razão disso, o paciente é encaminhado para uma das nove unidades referência da cidade, após a realização do tratamento do ferimento.

Do total de entrevistados, 93 (33,2\%) continuaram o esquema vacinal em um serviço diferente daquele onde iniciaram o tratamento. Entre esses, o motivo da troca mais relatado foi a proximidade da residência em 56 casos; 19,3\% devido à maior disponibilidade de horários de atendimen- 
Tabela 1. Distribuição dos casos de abandono do tratamento profilático antirrábico humano, segundo sexo, faixa etária, motivos da procura pelo atendimento e motivos da troca de serviço - Porto Alegre (RS), 2006.

\begin{tabular}{|c|c|c|}
\hline Variáveis & $\mathbf{n}$ & $\%$ \\
\hline \multicolumn{3}{|l|}{ Sexo } \\
\hline Masculino & 146 & 52,1 \\
\hline Feminino & 134 & 47,9 \\
\hline \multicolumn{3}{|l|}{ Faixa etária } \\
\hline 1 a 5 anos & 34 & 12,1 \\
\hline 6 a 12 anos & 52 & 18,6 \\
\hline 13 a 19 anos & 27 & 9,6 \\
\hline 20 a 59 anos & 142 & 50,8 \\
\hline$\geq 60$ anos & 25 & 8,9 \\
\hline \multicolumn{3}{|l|}{ Motivos da procura pelo atendimento } \\
\hline Tratamento do ferimento & 126 & 45,0 \\
\hline Vacinação antirrábica & 86 & 30,7 \\
\hline Gravidade da raiva & 39 & 14,0 \\
\hline Indicação de familiar, conhecido ou profissional de saúde & 11 & 3,9 \\
\hline Prevenção da doença & 6 & 2,1 \\
\hline Vacinação antitetânica & 5 & 1,8 \\
\hline Tratamento de problemas de saúde preexistentes & 4 & 1,4 \\
\hline Situações relacionadas a acidentes de trabalho & 3 & 1,1 \\
\hline Total & 280 & 100,0 \\
\hline \multicolumn{3}{|l|}{ Motivos da troca de serviço } \\
\hline Proximidade da residência & 56 & 60,2 \\
\hline Disponibilidade maior de atendimento & 18 & 19,3 \\
\hline Encaminhado pelo serviço do $1^{\circ}$ atendimento & 8 & 8,6 \\
\hline Administração do soro & 3 & 3,2 \\
\hline Ignorado & 3 & 3,2 \\
\hline Viagem & 2 & 2,2 \\
\hline Falta de vacina & 2 & 2,2 \\
\hline Vinculação anterior com o serviço & 1 & 1,1 \\
\hline Total & 93 & 100,0 \\
\hline
\end{tabular}

to; e $8,6 \%$ foram encaminhados pelo serviço que realizou o primeiro atendimento (Tabela 1 ).

Em relação à apresentação da carteira de vacina, apenas $28,2 \%(79 / 280)$ dos entrevistados apresentaram-na no momento da entrevista. Entre esses, verificou-se que, comprovadamente, $74,7 \%$ (59) haviam concluído o tratamento e 25,3\% (20) o interromperam. Entre os 201 casos que não apresentaram carteira, $127(63,2 \%)$ informaram haver concluído o tratamento, tendo citado, corretamente, o número de doses prescritas pelo serviço que realizou o primeiro atendimento. A partir dessas informações, verificouse que, dos 280 entrevistados, 66,4\% (186) concluíram o tratamento.

Quanto ao número de doses indicadas, para $2 / 3$ dos entrevistados foram prescritas duas doses. Desses, 69,8\% concluíram o tratamento. Entre os que receberam a indicação de cinco doses, apenas 59,3\% as realizaram (Tabela 2). Apesar da maior prevalência de interrupção entre os indivíduos com cinco doses indicadas, essa diferença não foi estatisticamente significativa $\left(\chi^{2}=\right.$ 3,03; $\mathrm{p}=0,08)$.

Foi testada, também, a associação entre sexo e abandono do esquema vacinal. O sexo feminino apresentou 31,3\% (42/134) de abandono, enquanto o masculino $35,6 \%$ (52/146), não sendo evidenciada diferença estatisticamente significativa $\left(\chi^{2}=0,57 ; p=0,45\right)$. O mesmo ocorreu em relação à faixa etária. Os indivíduos com menos de 13 anos apresentaram 33,7\% (29/57) de abandono, os adolescentes $51,9 \%(14 / 29)$ e os adultos $30,5 \%(51 / 167)\left(\chi^{2}=4,79 ; \mathrm{p}=0,09\right)$.

Quanto aos motivos do abandono, 24,5\% dos indivíduos relataram não terem considerado necessário completar o tratamento e 13,8\% não se sentiram adequadamente orientados sobre como dar continuidade a ele. Além desses, $10,6 \%$ referiram a falta de tempo para realiza- 
rem as vacinas ou a recomendação do profissional da saúde sobre a não necessidade de realizar a segunda dose se o animal estivesse saudável. As outras razões foram apontadas com menor frequência. Somente em 19,1\% dos casos houve a busca ativa dos faltosos pelos serviços de saúde (Tabela 3).

Quando perguntados sobre seu conhecimento sobre a importância da vacina, 70,7\% dos entrevistados referiram conhecer, indicando a prevenção da raiva como sua principal ação, seguida da prevenção de doenças em geral e da morte (Tabela 4).
Por fim, a avaliação da qualidade da indicação da vacina mostrou que, em 92,5\% (259/280) dos casos, ela foi indicada adequadamente e 7,5\% $(21 / 280)$ não necessitariam da vacina. Não se evidenciou associação estatisticamente significativa entre a qualidade da indicação e o abandono do esquema vacinal $\left(\chi^{2}=0,87 ; p=0,35\right)$.

\section{Discussão}

Uma das principais contribuições do presente estudo refere-se a seu ineditismo e, principalmen-

Tabela 2. Esquema vacinal indicado e número de vacinas realizadas - Porto Alegre (RS), 2006.

\begin{tabular}{|c|c|c|c|c|c|c|}
\hline \multirow{3}{*}{ Doses realizadas } & \multicolumn{4}{|c|}{ Esquema vacinal indicado } & & \\
\hline & \multicolumn{2}{|c|}{2 doses } & \multicolumn{2}{|c|}{5 doses } & \multicolumn{2}{|c|}{ Total } \\
\hline & $\mathbf{n}$ & $\%$ & $\mathbf{n}$ & $\%$ & $\mathbf{n}$ & $\%$ \\
\hline 1 & 57 & 30,2 & 10 & 11,0 & 67 & 23,9 \\
\hline 2 & 132 & 69,8 & 8 & 8,8 & 140 & 50,0 \\
\hline 3 & - & - & 9 & 9,9 & 9 & 3,2 \\
\hline 4 & - & - & 10 & 11,0 & 10 & 3,6 \\
\hline 5 & - & - & 54 & 59,3 & 54 & 19,3 \\
\hline Total & 189 & 67,5 & 91 & 32,5 & 280 & 100,0 \\
\hline
\end{tabular}

Tabela 3. Motivos da não conclusão do tratamento indicado - Porto Alegre (RS), 2006.

\begin{tabular}{llc}
\hline \multicolumn{1}{c}{ Motivos da não conclusão do tratamento } & n & $\%$ \\
\hline O paciente não considerou necessário completar o tratamento & 23 & 24,5 \\
Falta de orientação & 13 & 13,8 \\
Profissional da saúde recomendou que se o animal estivesse & 10 & 10,6 \\
saudável não era necessária a 2a dose vacinal & 10 & 10,6 \\
Falta de tempo & 8 & 8,5 \\
Dificuldades de atendimento & 6 & 6,4 \\
Esquecimento & 5 & 5,3 \\
Viagem & 4 & 4,3 \\
Dificuldade econômica para o transporte & 3 & 3,2 \\
Outros & 3 & 3,2 \\
Não quis ir & 2 & 2,1 \\
Preguiça & 2 & 2,2 \\
Vacina dolorida & 2 & 2,1 \\
Cicatrização do ferimento & 2 & 2,1 \\
Não respondeu & 1 & 1,1 \\
Dispensado pela Equipe de Zoonoses & & \\
Busca ativa pelo profissional de saúde & & 19,1 \\
Sim & 18 & $\mathbf{8 0 , 9}$ \\
Não & 76 & $\mathbf{1 0 0 , 0}$ \\
Total & $\mathbf{9 4}$ & \\
\hline
\end{tabular}




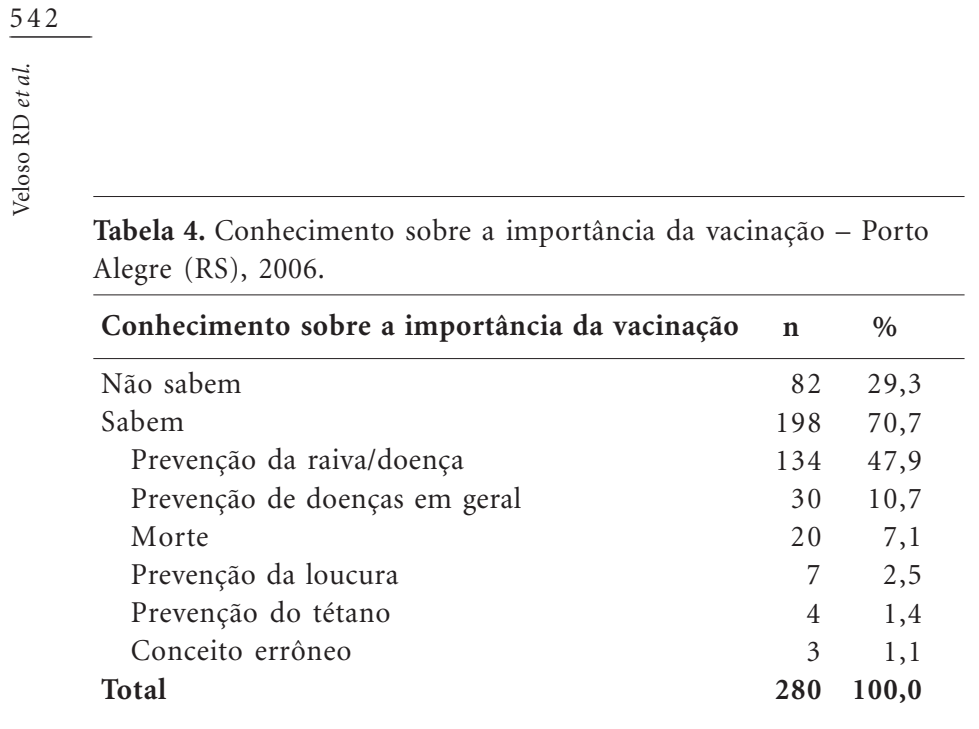

te, atualidade. Na revisão de literatura realizada, não foram encontrados estudos nacionais ou internacionais investigando o perfil de indivíduos que abandonaram o tratamento profilático com vacinas antirrábicas. Foi localizado, apenas, o estudo de Ribeiro Netto e Machado ${ }^{13}$, no final da década de 60, enfocando as razões do abandono. Com isso, a comparação dos achados do presente estudo ficou prejudicada, tendo sido realizada apenas em relação aos motivos da interrupção do tratamento.

Evidenciou-se alto percentual $(23,6 \%)$ de casos que necessitaram de substituição em razão de endereços não corretos. Isto é, na visita realizada ao endereço registrado no Sinan, a casa não foi localizada ou os seus moradores desconheciam a pessoa procurada. É possível que isso ocorra em razão de os serviços de saúde da capital serem buscados por moradores de outros municípios, e esses, com medo de não serem atendidos, informam endereços incorretos pertencentes a Porto Alegre. Por sua vez, o percentual de recusas $(3,2 \%)$ foi bastante reduzido, não comprometendo a representatividade da amostra.

Nesta pesquisa, evidenciou-se predomínio do sexo masculino e do grupo etário dos adultos, semelhante ao perfil de todos os atendimentos antirrábicos realizados no mesmo período em Porto Alegre e aos resultados encontrados por outros autores ${ }^{14,15}$.

Quase a metade dos indivíduos apontou como motivo da procura pelo atendimento o tratamento do ferimento. Isso pode indicar o desconhecimento sobre a gravidade da doença e também uma tendência de busca dos serviços para o tratamento de lesões mais graves, que necessi- tam de maiores cuidados. Menos de 1/3 referiram ser para receber a vacina contra a raiva, o que seria uma motivação adequada, em razão do risco da exposição. Alguns indivíduos referiram que a razão da busca era a vacinação antitetânica, realizada, quando necessário, em casos de mordeduras animais ${ }^{16}$. Embora o tratamento do ferimento e a vacinação antitetânica sejam procedimentos importantes, o principal motivo para a busca de atendimento por agressão animal deve ser a avaliação da situação para a indicação ou não da vacina e do soro.

Em relação aos pacientes com problemas de saúde preexistentes (diabetes, hipertensão, baixa imunidade), ainda que o principal motivo da procura de atendimento deva ser a prevenção da raiva, é necessário verificar se ocorreu o agravamento desses problemas, procedimento correto para a prevenção de possíveis complicações e a prescrição de cuidados especiais.

Os acidentes de trabalho também foram motivos considerados como adequados para a procura de atendimento. Nos casos em que a agressão animal ocorreu durante o trabalho, as empresas exigiram que o funcionário buscasse o serviço de saúde, mostrando preocupação com sua saúde e adequação à legislação trabalhista.

Idealmente, o paciente deveria iniciar e concluir a vacinação em um mesmo serviço, possibilitando assim seu melhor acompanhamento e evitando problemas de registro no Sinan. No entanto, por diferentes razões, ocorre a troca de serviço. A mais frequentemente relatada foi a distância, determinando busca por um serviço mais próximo à residência ou ao local de trabalho, o que facilita a conclusão do tratamento. Alguns entrevistados informaram terem trocado de serviço em busca de maior disponibilidade de horário para atendimento, em que ele seja oferecido diariamente, 24 horas por dia, inclusive sábados, domingos e feriados, de acordo com o preconizado pelas Normas Técnicas de Tratamento Profilático Antirrábico Humano ${ }^{1}$. Essa maior disponibilidade contribui para a continuidade do tratamento, sendo oferecida apenas em duas unidades referência do município (uma para atendimento de crianças até 12 anos e a outra para os maiores de 13 anos). O encaminhamento pelo serviço que realizou o primeiro atendimento também constitui-se em razão para a troca de serviço. Outras vezes, os pacientes atendidos necessitam de soro, sendo encaminhados às duas unidades referência que realizam esse procedimento. A centralização da disponibilidade do soro pode trazer algumas dificuldades para sua apli- 
cação, como o retardo no início de sua administração ou a desistência de sua busca pela dificuldade de locomoção.

Dois pacientes referiram ter trocado de serviço por falta de vacina, situação essa que não deveria acontecer, pelo risco de descontinuidade ao tratamento. Nenhum usuário informou descontentamento com o atendimento como motivo da troca de serviço.

Após as entrevistas, evidenciou-se que, dos 280 casos registrados no Sinan como abandono de tratamento, somente 94 haviam interrompido, de fato, a vacinação. A maioria dos indivíduos considerados como não tendo concluído o esquema vacinal indicado havia, na realidade, trocado de serviço para a realização da vacina. O serviço que deu continuidade não informou ao que iniciou o tratamento e preencheu a ficha de atendimento antirrábico do Sinan sobre a sua conclusão, assim como também não informou ao setor que coordena a vigilância da raiva humana na Secretaria Municipal de Saúde. Com isso, o sistema ficou desatualizado, sendo os casos interpretados, erroneamente, como abandono de tratamento.

Os resultados desta pesquisa nos levam a pensar que a taxa de abandono de tratamento, no segundo semestre de 2006, foi inferior aos $55,4 \%$ registrados no Sinan. Considerando-se que, entre os registrados como abandono, somente 33,6\% o eram de fato, pode-se aplicar essa mesma proporção nos 962, reduzindo-se este número para 323. Com isso, estima-se que a proporção de interrupção no total de atendimentos, no período estudado, seja de 14,5\%. É importante ressaltar que essa é apenas uma estimativa, devendo essa situação ser mais bem investigada em estudos posteriores.

Em Mato Grosso do Sull ${ }^{17}$, as Fichas de Atendimento Antirrábico foram informatizadas, resolvendo esse problema. O serviço de saúde, ao receber o paciente, abre a ficha de atendimento, realizando o primeiro registro, sendo os dados disponibilizados às outras unidades por meio de uma rede interligada. Esse registro pode ser acessado em qualquer unidade referência, possibilitando a inclusão de novos dados sobre o caso. Além disso, é disponibilizada a ficha vacinal na qual serão registradas as vacinas aplicadas.

A maioria dos entrevistados não dispunha da carteira de vacinação no momento da entrevista. No entanto, mesmo não tendo apresentado a carteira, os indivíduos que afirmaram haver concluído o tratamento relataram corretamente o número de doses prescritas, enquanto os que afirmaram terem abandonado o trata- mento também confirmaram corretamente o número de doses que deveriam ter realizado.

O percentual de abandono foi maior entre os que não tinham carteira do que entre os que tinham. Isso pode ser um indicador da baixa importância dada ao tratamento. Porém, essa diferença não foi estatisticamente significativa. Talvez isso se deva ao número de indivíduos avaliados, insuficiente para o estudo da associação, uma vez que a amostra não foi calculada para esse fim. Apesar disso, esse resultado pode indicar uma tendência.

$\mathrm{O}$ alto percentual de indivíduos sem carteira de vacina reforça a necessidade de os profissionais de saúde orientarem seus usuários sobre a importância do cuidado com esse documento.

O sistema informatizado de Mato Grosso do $\mathrm{Sul}^{17}$ também auxilia a solucionar o problema do extravio das carteiras de vacinação, permitindo melhor controle dessa situação.

Houve maior percentual de interrupção nos tratamentos com cinco doses indicadas. Esses são os casos mais graves e, ao mesmo tempo, apresentam maior risco de abandono por serem os de mais longa duração. Exigem do paciente maior disponibilidade de tempo, na medida em que ele necessita comparecer mais vezes ao serviço de saúde. Dependendo do caso, também podem trazer dificuldades econômicas, em razão de necessidade de transporte e maior chance de esquecimento da data aprazada.

O principal motivo da não conclusão do tratamento foi que os indivíduos não consideraram necessário completá-lo, isto é, consideraram sua indicação desnecessária. O paciente não deve abandonar ou interromper o tratamento antirrábico por conta própria, correndo o risco de contrair a doença pela falta do número necessário de doses para sua imunização ${ }^{9}$. O esquema vacinal indicado deve ser rigorosamente seguido ${ }^{3}$, sendo a interrupção do tratamento de responsabilidade exclusiva dos profissionais de saúde ${ }^{9}$.

Ribeiro Netto e Machado ${ }^{13}$ encontraram, como motivos do abandono, despesas de transporte, perdas de horas de trabalho e, no caso das crianças, a necessidade do acompanhamento de um responsável, além da falta de orientação dos profissionais da saúde sobre a importância do tratamento completo, semelhante ao encontrado no presente estudo.

Em relação ao item "outros" dos motivos do abandono, estão incluídos três diferentes motivos, cada um deles com um caso. Em um desses, o paciente referiu que interrompeu o tratamento por ter encontrado a carteira de vacinação do 
cão, porém, o histórico vacinal do animal não se constitui em fator suficiente para a dispensa do tratamento $^{1}$, pois um animal vacinado pode não estar imunizado, apesar de ter recebido a vacina. Outra paciente encontrava-se grávida, tendo ficado com receio de se vacinar. Entretanto, gravidez, doenças intercorrentes e outros tratamentos não representam contraindicação para a vacinação ${ }^{1}$. Essa situação demonstra que a paciente não foi suficientemente orientada pelo serviço de saúde. O terceiro paciente não desejava realizar o tratamento, tendo-o iniciado quando foi ao serviço de saúde procurar orientação sobre um procedimento cirúrgico e o médico constatou a presença de uma lesão por agressão animal, indicando a vacina. Nessa e em outras situações, é importante que o serviço de saúde informe sobre a gravidade da exposição ${ }^{6}$ para que o paciente se responsabilize por seu tratamento.

Em um caso, houve a liberação do tratamento pelo Centro de Controle de Zoonoses do município, procedimento de acordo com as normas técnicas do tratamento antirrábico, após a observação do animal por dez dias ou a realização de exame laboratorial. A observação do animal deve ser supervisionada por médico veterinário ou serviço municipal de vigilância da raiva em visita domiciliar ou no canil público ${ }^{3}$. No entanto, ainda que o procedimento estivesse correto, verificase a falta de integração entre o serviço médico e o médico veterinário, na medida em que o banco de dados do Sinan não foi retroalimentado.

Quanto às dificuldades no atendimento, foram citados motivos como: crianças desacompanhadas dos pais, falta de vacina no serviço, serviço fechado ou o paciente não haver levado sua carteira de vacinação. Todos esses motivos impossibilitaram a realização da vacina, contribuindo, no entendimento dos entrevistados, para o abandono do tratamento.

Somente em 19,1\% dos casos considerados pelo Sinan como abandono houve busca ativa pelo serviço de saúde, apesar de este ser responsável pela busca de faltosos na data aprazada para a aplicação das doses prescritas ${ }^{6}$. Essa situação indica a necessidade da sensibilização contínua da equipe de saúde sobre seu papel na busca ativa dos faltosos. Entretanto, sabe-se das dificuldades operacionais dos serviços para esse tipo de atuação, pela falta tanto de profissionais como de meio de transporte.

$\mathrm{O}$ alto percentual de indivíduos que referiram saber da importância da vacinação demonstra um bom nível de conhecimento por parte deles. Apesar disso, ações para divulgar infor- mações precisam ser implementadas para atingir a parcela desinformada. Nesse sentido, foi criado o Dia Mundial da Raiva, em 8 de setembro, com o objetivo de melhorar a conscientização sobre essa doença ${ }^{18}$. Nesse dia, em Porto Alegre, são realizadas várias atividades educativas para informar sobre as formas de transmissão, tratamento e prevenção da doença.

Em relação à qualidade do tratamento prescrito, verificou-se alto percentual de indicação adequada de vacinas (92,5\%), segundo as Normas Técnicas do Ministério da Saúde ${ }^{1}$. Como não foram encontrados outros artigos investigando essa situação entre casos de abandono, compararam-se os achados deste estudo com os de Rigo e Honer ${ }^{17}$, em Mato Grosso do Sul, e o de Moran et al. ${ }^{19}$ nos Estados Unidos, que investigaram a adequação da profilaxia antirrábica entre o total de casos atendidos.

No primeiro estudo, 41,9\% dos indivíduos atendidos em profilaxia antirrábica tiveram seu tratamento indicado adequadamente ${ }^{17}$. A situação identificada por Moran et al. ${ }^{19}$ foi mais favorável, sendo que $60 \%$ dos casos atendidos tiveram a indicação do tratamento considerada como adequada. Comparando esses resultados, verificou-se um percentual extremamente positivo de indicação correta de vacinação na população estudada. A ausência de associação entre a qualidade da indicação e o real abandono do tratamento parece sugerir que ela não possa ser considerada como um motivo para a interrupção do tratamento em Porto Alegre.

\section{Considerações finais}

$O$ alto percentual de abandono de tratamento identificado a partir do Sinan ocorreu devido a falhas na retroalimentação do sistema. Em razão disso, é necessário que os municípios viabilizem estratégias que permitam essa retroalimentação, quer informatizando o registro dos atendimentos, quer criando um fluxo em papel, quando do encerramento do esquema vacinal. No entanto, é importante ressaltar que, apesar de os problemas com o sistema terem representado $66,4 \%$ da taxa de abandono inicialmente detectada, a maioria dos casos confirmados como abandono estava relacionada à falta de entendimento dos pacientes quanto à importância do tratamento profilático. Nesse sentido, entendese que duas estratégias poderão ser utilizadas para seu enfrentamento: a capacitação da equipe de saúde e a educação em saúde da comunidade. 
A capacitação é necessária para a maior conscientização da equipe de saúde sobre a infecção humana, possibilitando a indicação adequada e oportuna da profilaxia, evitando que a prevenção da doença seja negligenciada ${ }^{19,20}$. Para tanto, é fundamental que os profissionais se sintam seguros para avaliar todos os aspectos envolvidos em cada caso: as características do ferimento, o estado de saúde do animal agressor, a possibilidade de observação para cães e gatos, a situação epidemiológica do local de origem do animal para a adequada prescrição do tratamento ${ }^{3}$, enfatizando a necessidade da conclusão do esquema vacinal prescrito. Além disso, é responsabilidade da equipe de saúde a busca ativa dos faltosos. No entanto, é necessário existirem recursos humanos e materiais para que isso aconteça.

As ações de educação em saúde devem ser desenvolvidas visando à responsabilização dos usuários por sua própria saúde. Elas se baseiam no estímulo à posse responsável de animais; castração e vacinação dos animais domésticos; na informação da população para o reconhecimento da gravidade da exposição às agressões animais, necessidade de atendimento imediato e medidas auxiliares que devem ser seguidas pelas pessoas expostas ou agredidas; e na identificação dos sintomas de um animal suspeito. Além disso, é importante divulgar os serviços de referência existentes; desmistificar o tratamento antirrábico humano e estimular a responsabilidade do paciente no cumprimento do esquema prescrito com vistas a reduzir o abandono e o risco da ocorrência da doença.

A situação encontrada no presente estudo aponta para a necessidade de outros estudos sobre o abandono do tratamento antirrábico humano, pois este é um tema pouco investigado, apesar de sua importância para a Saúde Coletiva.

\section{Colaboradores}

RD Veloso foi responsável pela concepção do estudo, coleta de dados e redação do artigo; DRGC Aerts participou da concepção do estudo, supervisão da coleta de dados e redação do artigo; LO Fetzer auxiliou na concepção do estudo e participou da redação final do artigo; CB dos Anjos realizou a revisão final do artigo; JC Sangiovanni participou da avaliação da qualidade do atendimento antirrábico humano e da revisão final do artigo. 


\section{Referências}

1. Brasil. Ministério da Saúde. Normas técnicas de tratamento profilático anti-rábico humano. Brasília: Ministério da Saúde; 2002.

2. Hankins DG, Rosekrans JA. Overview, prevention, and treatment of rabies. Mayo Clin Proc 2004; 79:671676.

3. Costa WA, Ávila CA, Valentine EJG, Reichmann MLAB, Cunha RS, Guidolin R, Panachão MRL, Omoto TM, Bolzan VL. Profilaxia da raiva humana. São Paulo: Instituto Pasteur; 2000. p. 33.

4. Acha PN, Szyfres B. Zoonosis y enfermedades transmisibles comunes al hombre y los animales. $2^{\mathrm{a}} \mathrm{ed}$. Washington: Organización Panamericana de la Salud; 1986. (Publicación Científica 503).

5. Rolim RLP, Lopes FMR, Navarro IT. Aspectos da vigilância epidemiológica da raiva no município de Jacarezinho, Paraná, Brasil, 2003. Semina: Ciências Agrárias 2006; 27(2):271-280.

6. Brasil. Ministério da Saúde. Secretaria de Vigilância em Saúde. Guia de Vigilância Epidemiológica. $6^{\mathrm{a}}$ ed. Brasília: Ministério da Saúde; 2005.

7. Centro Pan-Americano de Febre Aftosa. Eliminación de la rabia humana transmitida por perros en la América Latina: análisis de la situación, ano 2004. Washington, 2005. [acessado 2007 jan 9] [cerca de 20p.]. Disponível em: http://www.panaftosa.org.Br/ Comp/Zoonoses/Raiva/doc/POS_rabia_humana 2004.pdf

8. Warrel MJ. The challenge to provide affordable rabies postexposure treatment. Vaccine 2003; 21:706709.

9. Brasil. Ministério da Saúde. Educação em saúde na profilaxia da raiva. Brasília: Ministério da Saúde; 1981.

10. Costa WA. Aspectos práticos na prevenção da raiva humana. J Pediatr 1999; 75(Supl.1):135-148.

11. Garcia RCM, Vasconcellos SA, Sakamoto SM, Lopez AC. Análise de tratamento anti-rábico humano pós-exposição em região da Grande São Paulo, Brasil. Rev Saude Publica 1999; 33(3):295-301.

12. Deslandes SF, Cruz Neto O, Gomes R, Minayo MCS, organizadores. Pesquisa social: teoria, método e criatividade. Petrópolis (RJ): Vozes; 1994.
13. Ribeiro Netto A, Machado CG. Alguns aspectos epidemiológicos da exposição humana ao risco da infecção pelo vírus da raiva na cidade de São Paulo, Brasil. Rev Inst Med Trop 1970; 12(1):16-30.

14. Blanton JD, Bowden NY, Eidson M, Wyatt JD, Hanlon CA. Rabies postexposure prophylaxis, New York, 1995-2000. Emerg Infect Dis 2005; 11(12):1921-1927.

15. Alonso BPM. Estudo dos casos de agressões por cães no município de Araraquara, estado de São Paulo, Brasil [monografia]. Araraquara (SP): Universidade Estadual Paulista Júlio de Mesquita Filho; 2005.

16. Magnussen CR. Infecções da pele e dos tecidos moles. In: Reese RE, Douglas RG, editores. Doenças infecciosas. $2^{\mathrm{a}}$ ed. Rio de Janeiro: Medsi; 1989. p. 114-117.

17. Rigo L, Honer MR. Análise da profilaxia da raiva humana em Campo Grande, Mato Grosso do Sul, Brasil, em 2002. Cad Saude Publ 2005; 21(6):19391945.

18. Brasil. Ministério da Saúde. Dia Mundial da Raiva. [site na Internet]. 2006 [acessado 2007 set 8]. Disponível em: http://www.agricultura.gov.br/pls/portal/docs/PAGE/MAPA/PROGRAMA/AREA

19. Moran GJ, Talan DA, Mower W, Newdow M, Ong S, Nakase JY, Pinner RW, Childs JE. Appropriateness of rabies postexposure prophylaxis treatment for animal exposures. JAMA 2000; 284(8):1001-1007.

20. Rabies and Wildlife Zoonoses Group, Veterinary Laboratories Agency, WHO Collaborating Centre for the Characterization of Rabies and Rabies-Related Viruses, UK. Rabies remains a "neglected disease”. Euro Surveill 2005; 10:10-12.

Artigo apresentado em 11/09/2008

Aprovado em 08/06/2009

Versão final apresentada em 09/07/2009 